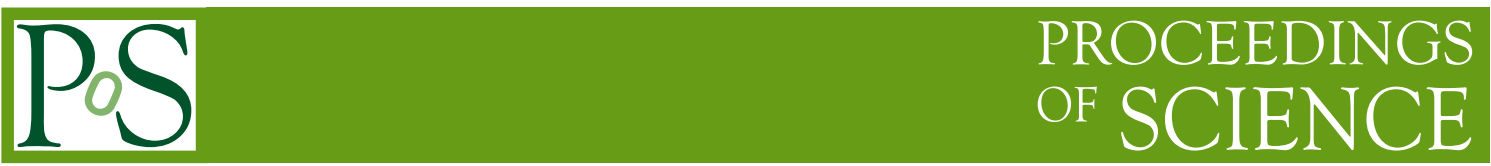

\title{
Distances to supernovae: an efficient probe of dark energy
}

Pierre Astier*

LPNHE/CNRS/UPMC

E-mail: pierre.astier@in2p3.fr

Measuring distances to supernovae (or more precisely to the "type Ia" subclass) has allowed us to measure the distance-redshift relation beyond linear order for the first time in 1998. These first results already showed strong evidence for an accelerated cosmological expansion at the present epoch. This has been confirmed by several other cosmological probes, but what causes this accelerated expansion remains elusive. Our ignorance is commonly parametrized using the "equation of state of dark energy", where dark energy refers to the fluid one can postulate to source the acceleration. I will present how supernova measurements have evolved since the discovery, review the latest dark energy constraints, and discuss the future of the probe.

Frontiers of Fundamental Physics 14 - FFP14,

15-18 July 2014

Aix Marseille University (AMU) Saint-Charles Campus, Marseille

${ }^{*}$ Speaker. 